\title{
THE CLUSTER OF BLUE STARS SURROUNDING THE M31 NUCLEAR BLACK HOLE*
}

\author{
Tod R. Lauer ${ }^{1}$, Ralf Bender ${ }^{2,3}$, John Kormendy ${ }^{2,3,4}$, Philip Rosenfield ${ }^{5}$, and Richard F. Green ${ }^{6}$ \\ ${ }^{1}$ National Optical Astronomy Observatory, P.O. Box 26732, Tucson, AZ 85726, USA \\ ${ }^{2}$ Universitäts-Sternwarte München, Ludwig-Maximilians-Universität, Scheinerstrasse 1, München D-81679, Germany \\ ${ }^{3}$ Max-Planck-Institut für Extraterrestrische Physik, Giessenbachstrasse, D-85748 Garching, Germany \\ ${ }^{4}$ Department of Astronomy, University of Texas at Austin, 1 University Station C1400, Austin, TX 78712-0259, USA \\ ${ }^{5}$ Astronomy Department, University of Washington, Seattle, WA 98195, USA \\ ${ }^{6}$ Large Binocular Telescope Observatory, University of Arizona, 933 North Cherry Avenue, Tucson, AZ 85721, USA \\ Received 2011 October 3; accepted 2011 December 5; published 2012 January 6
}

\begin{abstract}
We obtained $U_{330^{-}}$and $B$-band images of the M31 nucleus using the High Resolution Camera of the Advanced Camera for Surveys on board the Hubble Space Telescope (HST). The spatial resolution in the $U_{330}$ band, $0^{\prime} 03$ FWHM, or 0.1 pc at M31, is sufficient to resolve the outskirts of the compact cluster (P3) of UV-bright stars surrounding the M31 black hole. The center of the cluster is marked by an extended source that is both brighter and redder than the other point sources within P3; it is likely to be a blend of several bright stars. We hypothesize that it marks the location of the M31 black hole. Both stellar photometry and a surface brightness fluctuation analysis show that the P3 stellar population is consistent with early-type main-sequence stars formed in a 100-200 Myr old starburst population. Evolutionary tracks of post early asymptotic giant branch (PEAGB) stars, associated with late-stage evolution of an old population, also traverse the $U$ and $U-B$ domain occupied by the P3 stars; but we argue that only a few stars could be accounted for that way. PEAGB evolution is very rapid, and there is no progenitor population of red giants associated with $\mathrm{P} 3$. The result that $\mathrm{P} 3$ comprises young stars is consistent with inferences from earlier HST observations of the integrated light of the cluster. Like the Milky Way, M31 harbors a black hole closely surrounded by apparently young stars.
\end{abstract}

Key words: galaxies: individual (M31) - galaxies: nuclei

Online-only material: color figures

\section{BLUE STARS/BLACK HOLE}

There is a highly compact cluster of blue stars (Lauer et al. 1998; Brown et al. 1998) at the heart of the M31 double nuclear cluster of old stars. Kormendy \& Bender (1999) showed that this blue cluster in turn hosts the $\sim 10^{8} M_{\odot}$ supermassive black hole also known to reside in the nucleus (Dressler 1984; Dressler \& Richstone 1988; Kormendy 1988; Richstone et al. 1990). Bender et al. (2005) argue that the integrated spectrum and spectral energy distribution (SED) of the stars are consistent with their formation in a burst $200 \mathrm{Myr}$ ago. Young stars are also closely bound to the nuclear black hole in our Galaxy (Forrest et al. 1987; Allen et al. 1990; Krabbe et al. 1995), suggesting that this may not be a rare phenomenon. Understanding how apparently young stars were formed deeply interior to the tidal field of a supermassive black hole probes several issues of how stars and gas interact within such an extreme environment (see Alexander 2006 for a review). Study of the blue cluster in M31 provides an additional context in which to test theories for the formation of the unusual population surrounding the Milky Way black hole.

There is a diversity of ideas for explaining the origin of the M31 blue cluster. Chang et al. (2007), for example, argue that the cluster is the expected consequence of the non-axisymmetric structure of the surrounding M31 nucleus funneling gas from stellar mass loss into orbit around the black hole, where it periodically reaches surface densities sufficient to induce collapse and star formation. Likewise, Levin \& Beloborodov

\footnotetext{
* Based on observations made with the NASA/ESA Hubble Space Telescope, obtained at the Space Telescope Science Institute, which is operated by the Association of Universities for Research in Astronomy, Inc., under NASA contract NAS 5-26555. These observations are associated with GO 10571.
}

(2003), Bonnell \& Rice (2008), and Wardle \& Yusef-Zadeh (2012) have advanced idea of the formation of compact disks of young stars around the Milky Way black hole by massive central accretion of gas. In contrast, Demarque \& Virani (2007) argue that the blue stars in M31 result from stellar collisions and tidal stripping acting on an old population of stars interacting within the high-velocity orbits and strong tidal field associated with the black hole. We present new observations of the blue cluster obtained at the maximum angular resolution offered by the Hubble Space Telescope (HST) to provide better constraints on the origin of the stars residing in this unusual environment.

\subsection{The Discovery of the Blue Cluster}

The discovery of the blue cluster proceeded in stages. King et al. (1995) imaged the nucleus of M31 with the HST Faint Object Camera at $1750 \AA$, seeing a double structure similar to that discovered in the optical by Lauer et al. (1993). By the simple expedient of checking the position angle of their images, however, they discovered that the optically dimmer peak, designated P2 by Lauer et al., was actually brighter in the UV than P1. King et al. (1995) lacked a signal-to-noise ratio sufficient to resolve the spatial structure of the UV source at P2, noting only that it was highly compact and possibly consistent with a point source, such as low-level emission associated with the weak AGN detected in the radio (Crane et al. 1992), or emission from a single post-asymptotic giant branch (PAGB) star, such as those seen further out from the nucleus (King et al. 1992; Bertola et al. 1995). In retrospect, the discovery of a UV-bright nuclear source explains the observations of Nieto et al. (1986), who found the nucleus to have a color gradient becoming bluer toward its center, using Canada-France-Hawaii 
Telescope images obtained at $3750 \AA$ A Discovery of the cluster may also have been presaged by an enigmatic reference in Redman \& Shirley (1937) to third-party unpublished "Mt. Wilson material" purporting to show that "the spectrum of the nucleus is of a peculiar dwarf A type," a result supported by no other work prior to Bender et al. (2005) to the best of our knowledge from a literature search. ${ }^{7}$

Later HST observations by Lauer et al. (1998) and Brown et al. (1998) were able to resolve the UV source; both papers argued that it is a cluster of stars. WFPC2/PC images at $3000 \AA$ obtained by Lauer et al. (1998) showed that the half-power radius of the blue source is $\sim 0.2 \mathrm{pc}$. Lauer et al. (1998) further combined their $U$ - and $V$-band fluxes of the source with the King et al. (1995) fluxes at $1750 \AA$ to conclude that the source is consistent with an A-star SED.

Bender et al. (2005) refined this picture further by obtaining HST STIS spectroscopy of the nucleus over $\lambda \approx 3600-5100 \AA$ and reanalyzing the F300W images presented in Lauer et al. (1998). The spectroscopy shows strong Balmer lines, including a strong Balmer break, consistent with either $\mathrm{A} 0$ main-sequence (MS) or giant stars, but not a population of white dwarfs of the same photospheric temperature. The best match to the spectrum is provided by a $200 \pm 50 \mathrm{Myr}$ population formed in a single burst. Populations of less than half this age would exhibit too much UV flux to the blue side of the Balmer break.

Bender et al. (2005) further show that the stars are distributed in a flat disk with an exponential profile of a scale length of $0.37 \pm 0.04 \mathrm{pc}$ in rapid rotation about the black hole. ${ }^{8}$ The rotational broadening of the STIS spectrum implies a black hole mass of $M_{\bullet}=1.4 \times 10^{8} M_{\odot}$, a significant upward revision from earlier determinations.

\subsection{The Blue Cluster in the Context of the M31 Nucleus}

The M31 black hole and its cluster of blue stars mark the center of a much larger nuclear star cluster of complex structure. The surface brightness of the nuclear stellar system begins to rise above the underlying M31 bulge interior to $r \sim 3^{\prime \prime}$ or $\sim 10 \mathrm{pc}$ from the center. The cluster becomes increasingly elliptical at smaller radii, with $1-b / a>0.3$ for $r<1^{\prime \prime} .7$, but its isophotes remain concentric with the photo-center of the bulge for $r>1$."4 (Lauer et al. 1993). At yet smaller radii the nuclear cluster exhibits the double-peaked structure discovered by Lauer et al. (1993). The optically brighter peak, P1, is offset by 0'49 from the secondary brightness peak, P2, and a slightly lesser amount from the M31 bulge photo-center.

The best explanation for the double morphology is that both peaks result from the line-of-sight projection of an eccentric disk of stars roughly $\sim 2 \mathrm{pc}$ in diameter bound to the black hole (Tremaine 1995). The black hole strongly dominates the potential, thus the stars in the disk follow Keplerian orbits, spending most of their time at the orbital apocenter, creating an enhancement of light at P1. Significantly, the P1-P2 line twists away from the major axis of the outer nucleus by $\sim 20^{\circ}$. P1 and $\mathrm{P} 2$ are both redder in $V-I$ than the surrounding nucleus (Lauer et al. 1998). High spatial resolution spectroscopy shows that both P1 and P2 comprise old stellar populations with characteristics more like each other, than the underlying bulge

\footnotetext{
7 We have also been unable to find photographic spectra of the M31 nucleus prior to 1937 in catalogs of Mt. Wilson observations. We thank Dr. Christopher Burns of the Carnegie Observatories for searching for this material.

8 We use $770 \mathrm{kpc}$ as the distance to M31 (Freedman \& Madore 1990).
}

(Kormendy \& Bender 1999). The "Tremaine disk" thus appears to be a distinct component embedded in a much larger nuclear cluster. The initial model of the disk provided by Tremaine (1995) has been refined by the more detailed analysis presented by Peiris \& Tremaine (2003). An interesting feature of the refined model is that the disk requires a central hole in order to generate the apparent minimum of stellar emission that occurs between $\mathrm{P} 1$ and $\mathrm{P} 2$.

The cluster of blue stars occurs at still smaller radii, $r<0$ '. 1 , and must be closely bound to the black hole, which was first inferred to exist from ground-based spectroscopy (Dressler 1984; Dressler \& Richstone 1988; Kormendy 1988; Richstone et al. 1990). Kormendy \& Bender (1999) carefully registered their high spatial resolution spectroscopy to the WFPC2 imagery of Lauer et al. (1998), concluding that the M31 black hole was coincident with the cluster and that both were within 0 '.07 of the M31 bulge photo-center. The conclusion that the cluster hosts the black hole was further confirmed by Bender et al. (2005), who used STIS spectroscopy to show that the central velocity dispersion in the cluster rises to $1183 \pm 200 \mathrm{~km} \mathrm{~s}^{-1}$, with organized rotation peaking at $618 \pm 81 \mathrm{~km} \mathrm{~s}^{-1}$. Building on the analysis of Kormendy \& Bender (1999), they reduced the offset of the black hole from the bulge center to 0 '’033 in the "anti-P1" direction along the P1-P2 line. They further demonstrated that this slight offset is balanced by the asymmetry of the Tremaine disk - the center of mass of the complete nuclear system is coincident with the bulge photo-center to $<0^{\prime} \cdot 01$. Bender et al. (2005) also designated the cluster as a third component, P3, of the inner nucleus. This designation includes a subtle redefinition of P2 as it previously had been introduced by Lauer et al. (1993) and used by Lauer et al. (1998). The center of the cluster is coincident with the peak of a shallow surface brightness cusp seen in $V$ band that falls within 0 '. 1 of the bulge center; it was this location that the two Lauer et al. papers adopted as the center of P2. Bender et al. (2005), however, use P2 to denote the elongated and more diffuse concentration of older stars that extends from the cluster on the side opposite from P1. In this schema, P1 and P2 explicitly correspond to the apocenter and pericenter apses of the Tremaine disk, which has a mean pericenter that is not coincident with the cluster.

The present environment of $\mathrm{P} 3$ appears to be largely quiescent, despite the immediate proximity of the black hole (see the extensive discussion of this topic by Li et al. 2009). Crane et al. (1992) find a weak radio source coincident with P3, and Garcia et al. $(2005,2010)$ find low-level X-ray emission associated with P3, as well (however, see Li et al. 2009). On the other hand, a high spatial resolution map of emission from ionized gas within in the nucleus finds a few sources within an arcsecond of P1, but nothing coincident with P2 or P3 (del Burgo et al. 2000). While central accretion of gas may have created the P3 stars in the past, there is no evidence that any significant reservoir of cold gas exists there at present.

\subsection{A Closer Look...}

Under the Bender et al. (2005) description of P3, the cluster is a disk comprising only a few hundred A-stars. Given the scale length of the disk, we concluded that it should begin to show resolution into discrete sources in images with slightly higher angular resolution than were obtained by Lauer et al. (1998), such as could be provided by the HST Advanced Camera for Surveys (ACS)/High Resolution Channel (HRC) in the blue. The photometry of the brighter sources might be obtained directly, but in any case, the full image of the cluster 


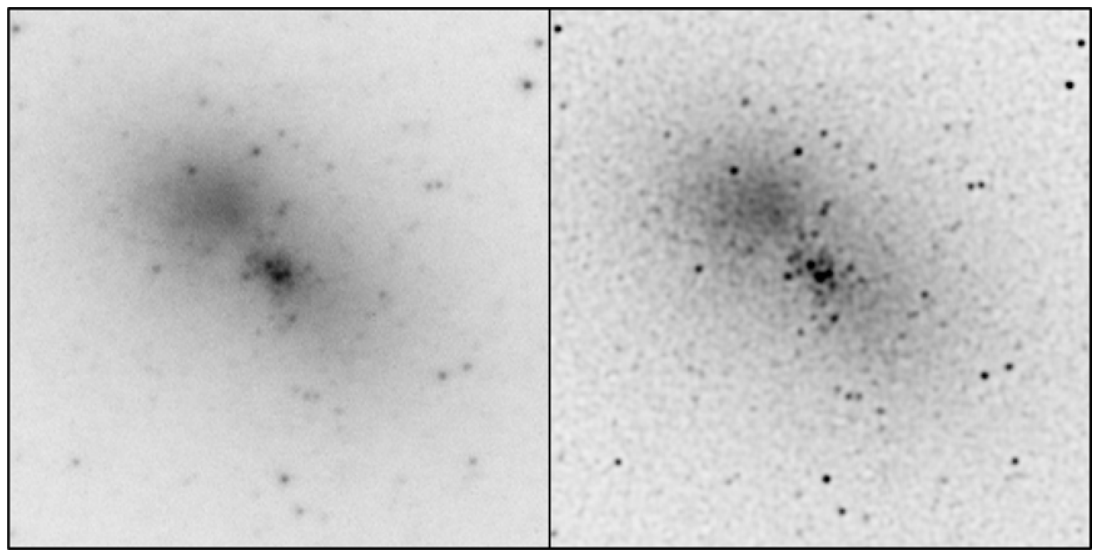

Figure 1. Central 3". $00 \times 33^{\prime \prime} 00$ of the $U_{330}(\mathrm{~F} 330 \mathrm{~W}) \mathrm{ACS} / \mathrm{HRC}$ image of the M31 nucleus is shown before (left) and after (right) deconvolution. North is at the top, and east to the left. The P3 cluster is at the center of the two panels. P1 is the diffuse concentration of starlight toward the NE, while P2 is the less prominent extension of light to the SW. Note that the area shown is still within the overall nuclear cluster. The M31 bulge only dominates at still larger radii.

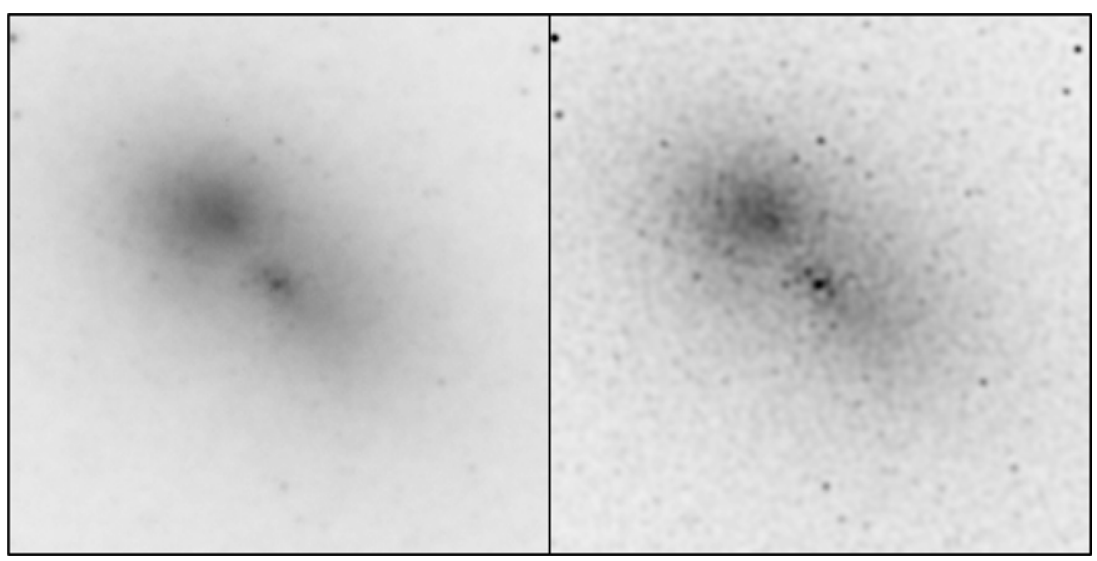

Figure 2. As in Figure 1, but for the $B(\mathrm{~F} 435 \mathrm{~W})$ ACS/HRC image instead.

could also be analyzed by surface brightness fluctuation (SBF) analysis (Tonry \& Schneider 1988) to constrain the P3 stellar population. If the cluster indeed comprises MS A-stars, it would look "lumpy" in HRC images. SBF could quantify the amplitude of the lumpiness, and in turn the typical luminosity of the stars that the cluster comprises.

\section{OBTAINING THE HIGHEST SPATIAL RESOLUTION IMAGE OF THE NUCLEUS}

We obtained images of the M31 nucleus under GO program 10571, using the HST ACS/HRC, which provided the best angular resolution available on the telescope. Images were obtained in filters F330W $\left(U_{330}\right)^{9}$ and F435W $(B)$ to best enhance the visibility of the blue cluster against the contrast of the older, redder population of the larger surrounding nucleus. Even the fine scale of the HRC $\left(0{ }^{\prime} .028 \times 0\right.$.'025) undersamples the point-spread function (PSF) in the blue, however; we thus dithered the exposures in a $2 \times 2$ square pattern of 0.5 pixel substeps (respecting the different angular sampling pitches of the two $C C D$ axes). In detail, the $B$-band images were obtained in a single orbit comprising two dither sets with a total of eight $298 \mathrm{~s}$ exposures. The two sets were offset by 3.5 pixels to reject hot pixels and other fixed defects. The reduced flux and sensitivity of the camera in the $U_{330}$ band required three orbits to obtain

\footnotetext{
9 We have designated the F330W filter bandpass as " $U_{330}$ " to avoid confusing it with the standard Johnson $U$ filter, which has a significantly different passband. All magnitudes are on the Vega system, except as noted.
}

an adequate signal. A complete dither set was obtained in each orbit for a total of twelve $672 \mathrm{~s}$ exposures. The three sets were offset from each other in an L-shaped pattern of 0.'125 steps, again to reject CCD defects. The total peak signal levels per pixel are $\sim 3100 e^{-}$in $\mathrm{P} 3$ and $\sim 1600 e^{-}$in $\mathrm{P} 1$ for the $U_{330}$ image; and $\sim 8400 e^{-}$in P3 and $\sim 7600 e^{-}$in P1 for the $B$ image.

The goal of the image reduction was to produce a single summed image for each filter with a $2 \times$ finer scale, sufficient to ensure Nyquist sampling. Because the images were obtained with non-redundant pointings, the image construction was done in stages to eliminate cosmic-ray events and other defects. The sub-pixel dither patterns were executed very precisely, thus each dither set could be simply interlaced to produce an initial upsampled image at the given pointing, with the images within the set used to recognize and repair (with interpolation) cosmic-ray events by comparing any one image to its three neighbors. The two $B$ and three $U_{330}$ interlaced images were then combined into a single image for each filter. This initial estimate of a Nyquist-sampled image was then used in a repeat of the first reduction cycle to go back to and improve the initial detection and repair of cosmic-ray events (and fixed CCD defects) in the source images.

With the second cycle of defect repair completed, the source images could be then combined more precisely to make a Nyquist-sampled image directly, using the Fourier method of Lauer (1999). This procedure combines the images in the Fourier domain to algebraically remove the aliased power present in the source images; significantly, it handles departures from 
Table 1

Sources in the Central Parsec of M31

\begin{tabular}{|c|c|c|c|c|c|c|c|}
\hline No. & $\begin{array}{c}\Delta \alpha \\
(\operatorname{arcsec})\end{array}$ & $\begin{array}{c}\Delta \delta \\
(\operatorname{arcsec})\end{array}$ & $m_{330}$ & $M_{330}$ & $m_{330}-m_{435}$ & $M_{U}$ & $U-B$ \\
\hline S01 & -0.225 & -0.022 & $22.82 \pm 0.15$ & -2.05 & $-1.10 \pm 0.48$ & -1.61 & -0.65 \\
\hline $\mathrm{S} 02$ & -0.164 & 0.045 & $22.54 \pm 0.12$ & -2.33 & $-0.93 \pm 0.34$ & -1.96 & -0.53 \\
\hline S03 & -0.121 & 0.003 & $22.85 \pm 0.16$ & -2.02 & $-0.20 \pm 0.28$ & -1.88 & -0.05 \\
\hline S04 & -0.079 & -0.237 & $22.22 \pm 0.09$ & -2.65 & $-0.86 \pm 0.25$ & -2.30 & -0.49 \\
\hline S05 & -0.064 & -0.045 & $22.68 \pm 0.14$ & -2.19 & $0.14 \pm 0.20$ & -2.06 & 0.24 \\
\hline S06 & -0.061 & 0.061 & $22.75 \pm 0.14$ & -2.12 & $-0.50 \pm 0.30$ & -1.89 & -0.25 \\
\hline S07 & -0.047 & 0.016 & $21.67 \pm 0.06$ & -3.20 & $-0.66 \pm 0.13$ & -2.92 & -0.36 \\
\hline S08 & -0.014 & -0.158 & $23.06 \pm 0.19$ & -1.81 & $-1.67 \pm 0.82$ & -1.16 & -1.00 \\
\hline S09 & -0.005 & 0.072 & $22.43 \pm 0.11$ & -2.44 & $-1.29 \pm 0.40$ & -1.94 & -0.77 \\
\hline S10 & -0.005 & 0.128 & $23.27 \pm 0.23$ & -1.60 & $-0.74 \pm 0.53$ & -1.29 & -0.42 \\
\hline $\mathrm{S} 11$ & 0.000 & 0.000 & $21.05 \pm 0.03$ & -3.82 & $0.27 \pm 0.04$ & -3.71 & 0.36 \\
\hline $\mathrm{S} 12$ & 0.005 & -0.092 & $22.66 \pm 0.13$ & -2.21 & $-0.96 \pm 0.38$ & -1.82 & -0.56 \\
\hline $\mathrm{S} 13$ & 0.029 & 0.235 & $22.93 \pm 0.17$ & -1.94 & $-1.32 \pm 0.60$ & -1.42 & -0.79 \\
\hline S14 & 0.038 & -0.231 & $22.92 \pm 0.17$ & -1.95 & $-0.66 \pm 0.39$ & -1.68 & -0.36 \\
\hline $\mathrm{S} 15$ & 0.052 & 0.063 & $21.60 \pm 0.05$ & -3.27 & $-0.64 \pm 0.12$ & -3.00 & -0.35 \\
\hline S16 & 0.103 & 0.212 & $22.84 \pm 0.16$ & -2.03 & $-1.01 \pm 0.46$ & -1.64 & -0.59 \\
\hline S17 & 0.114 & 0.136 & $22.78 \pm 0.15$ & -2.09 & $-0.58 \pm 0.33$ & -1.84 & -0.31 \\
\hline S18 & 0.120 & 0.027 & $22.62 \pm 0.13$ & -2.25 & $-0.27 \pm 0.24$ & -2.10 & -0.09 \\
\hline S19 & 0.169 & 0.092 & $22.79 \pm 0.15$ & -2.08 & $-0.93 \pm 0.42$ & -1.71 & -0.54 \\
\hline S20 & 0.177 & -0.005 & $22.24 \pm 0.09$ & -2.63 & $-0.51 \pm 0.20$ & -2.40 & -0.26 \\
\hline
\end{tabular}

Notes. The $m_{330}$ values are not corrected for galactic extinction, while all values in the subsequent columns are corrected assuming $A_{U}=0.44 \mathrm{mag}$, and $A_{B}=0.36 \mathrm{mag}$. Standard Johnson $U$ and $B$ values are derived using the color transformations in Sirianni et al. (2005). The distance to M31 is assumed to be $770 \mathrm{kpc}$ (Freedman \& Madore 1990). S07 and S11 are extended and are probably blends of several stars. S11 is hypothesized to be the location of the M31 black hole.

the optimal dither pattern and combines the complete data set into the optimal average Nyquist-sampled image (in a leastsquares sense). Crucially, the Lauer (1999) method of image reconstruction is used in preference to Drizzle (Fruchter \& Hook 2002). The Fourier method requires no parametric choices and avoids the blurring inherent in Drizzle reconstructions.

The final reduction step for the $U_{330}$ and $B$ images was to reproject them to rectilinear sampling, removing the inherent angular shear and the rectangular $0^{\prime \prime} .028 \times 00^{\prime} .025$ pixels present in the native HRC image sampling. This was done with reference to the STScI field distortion tables for the HRC, which vary with filter. Sinc-function interpolation was used to compute the undistorted images. This is the only appropriate interpolation algorithm to use for Nyquist-sampled images; it does not degrade the resolution in any way. The final pixel scale of the images was selected to be $0{ }^{\prime} \cdot 0114$, which is $1 / 4$ of the WFPC2/PC pixel scale, to allow for convenient comparison of the present HRC images to the $V$ and $I$ M31 nucleus images presented in Lauer et al. (1998). The Nyquist-sampled, rectified, and summed $U_{330}$ and $B$ images of the M31 nucleus are presented in Figures 1 and 2.

The spatial resolution of the $U_{330}$ images is $0^{\prime} .039$ FWHM, or $0.15 \mathrm{pc}$ at M31, putting this among the highest resolution images obtained by HST. As sharp as this PSF width is, however, it is actually significantly broader than a purely diffraction-limited PSF at $330 \mathrm{~nm}$, which has 0'.029 FWHM. This is due in part to the blurring associated with the HRC pixel kernel; however, PSF deconvolution can largely correct for this, as well removing the wings of the PSF, yielding nearly diffraction-limited images at both $U_{330}$ and B. Figures 1 and 2 also show the nuclear images after 40 iterations of Lucy-Richardson (Lucy 1974; Richardson 1972) deconvolution. The PSFs required were generated from bright stars present in the outskirts of the images. Tests presented in Lauer et al. (1998) show that this deconvolution procedure is well suited to recovering the intrinsic light distribution of the M31 nucleus. The final FWHM after deconvolution is $0^{\prime \prime} 030$, which corresponds to a physical resolution at $\mathrm{M} 31$ of $0.11 \mathrm{pc}$.

\section{THE STRUCTURE AND STELLAR POPULATION OF THE BLUE CLUSTER}

The UV-bright P3 star cluster clearly dominates the $U_{330}$ image. The cluster has a clumpy appearance in this filter, and further breaks up into apparently discrete sources in its outskirts (about $>0$.'05 from the center of the cluster). P3 is still bright in the $B$ image; however, it appears to be less clumpy and more concentrated. As we show below, the source that appears to define the center of P3 (S11 in Table 1) is somewhat redder than the surrounding cluster, while the discrete sources in the cluster outskirts are especially blue. The center of P3 thus increases in relative prominence going from the $U_{330}$ to the $B$ bandpass. The center of P3 also remains unresolved. S11 and a secondary maximum (S07 in Table 1) only offset from it by 0.'05 are both extended rather than stellar. Both sources likely comprise the blended light of several bright stars.

At large radii, both the $U_{330}$ and $B$ images show the presence of a number of hot stars distributed throughout the nucleus. ${ }^{10}$ This is the UV-bright stellar population observed by King et al. (1995) and Brown et al. (1998), although these authors did not characterize sources within 1 1.5 of the nucleus. The visual impression is that the hot stars outside of P3 appear to be weakly concentrated about the cluster, but show no obvious correlation with the $\mathrm{P} 1$ or $\mathrm{P} 2$ concentrations of the Tremaine disk.

$\mathrm{P} 3$ is much less prominent at redder bandpasses. It is essentially invisible at $I$ band, and shows up only weakly in $V$ band, as can be seen in Figure 3, which compares the present

\footnotetext{
10 As noted above, the nucleus dominates the center of M32 for $r<3^{\prime \prime}$. All the images shown in this paper are limited to radii interior to the nucleus.
} 


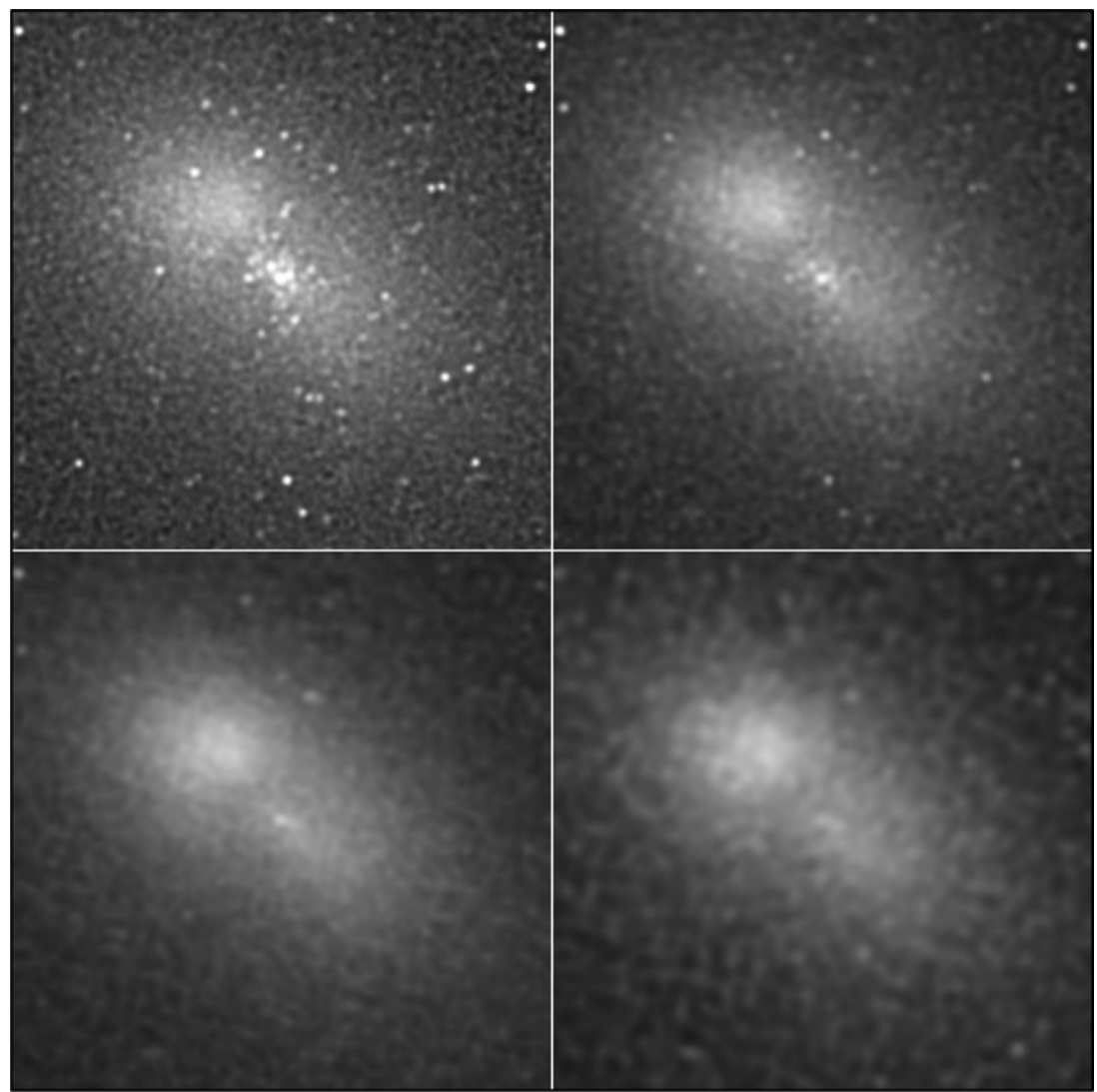

Figure 3. The prominence of the $\mathrm{P} 3$ cluster of blue stars as a function of wavelength is evident in this montage showing images of the M31 nucleus in four different bands. The upper left panel is the $U_{330} \mathrm{HRC}$ image, the upper right panel is the $B$ HRC image, while the lower left and right panels are the $V$ - and $I$-band WFPC2/PC images presented in Lauer et al. (1998). All images are deconvolved and normalized to have the same relative surface brightness at the center of the diffuse P1 component. While the blue cluster dominates the image in $U_{330}$, it is essentially invisible at $I$. Each panel is $3^{\prime \prime} .00 \times 33^{\prime \prime} 00$. North is at the top, and east to the left.

HRC images to the WFPC2/PC images presented in Lauer et al. (1998). For this comparison, all four images were normalized to have the same surface brightness at the center of the diffuse P1 component. The WFPC2/PC images had already been subsampled to a scale $2 \times$ finer than that of the native PC scale, and were readily shifted, interpolated, and rotated using sinc-function interpolation to a final scale $4 \times$ finer than the native PC scale. Figure 4 shows the $U_{330}, B$, and $V$ images combined to make a representative color image. The reddish color of the Tremaine disk contrasts strongly with the hot blue P3 population. In passing, we note that the grainy texture of $\mathrm{P} 1, \mathrm{P} 2$, and the surrounding nucleus in the deconvolved images is due to strong intrinsic SBF. Close comparison of the $B$ and $V$ images shows them to have correlated SBF patterns, an impressive agreement, given that the images were taken with different HST cameras, with differing PSFs and pixel scales. At 0 '.03 angular resolution, the image "noise" characteristics are dominated by the finite number of stars making up the nuclear components, not the number of photons collected by the instruments.

The reduced visibility of $\mathrm{P} 3$ at $V$ and $I$ highlights the distinction between it and P2, under the revised schema of Bender et al. (2005). P2, which would now be considered to be the stellar emission associated with the pericenter portion of the Tremaine disk, is visible in the $V$ and $I$ images as a highly elongated feature extending beyond P3 in the "anti-P1" direction. The centroid of this emission is clearly displaced from P3. Figures 1 and 2 are shown as negative prints, while Figure 3 is given a positive gray scale to offer some diversity in representing the inner nucleus. The negative gray scale in the

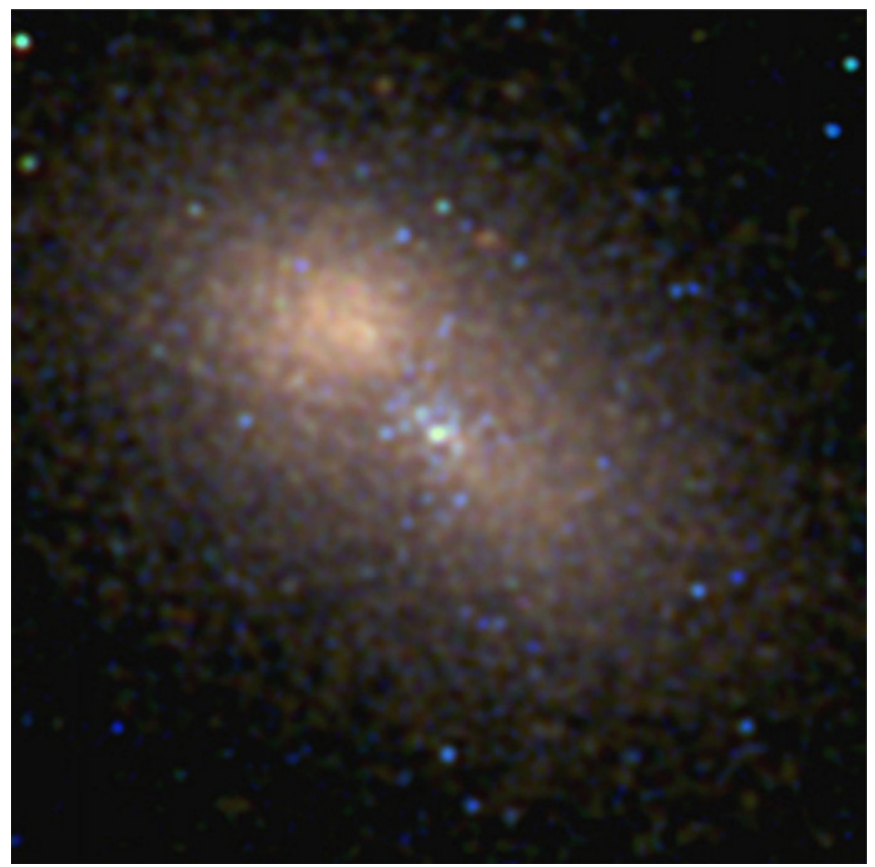

Figure 4. $V-, B$-, and $U_{330}$-band deconvolved images of the M31 nucleus are combined as an RGB triplet in an attempt to generate a plausible "real color" image of the nucleus. The scale and orientation are as in Figures 1-3. The graininess of the image is due to the SBF pattern intrinsic to the nucleus.

first two figures, for example, appears to accentuate the diffuse toroidal morphology of the Tremaine disk. 


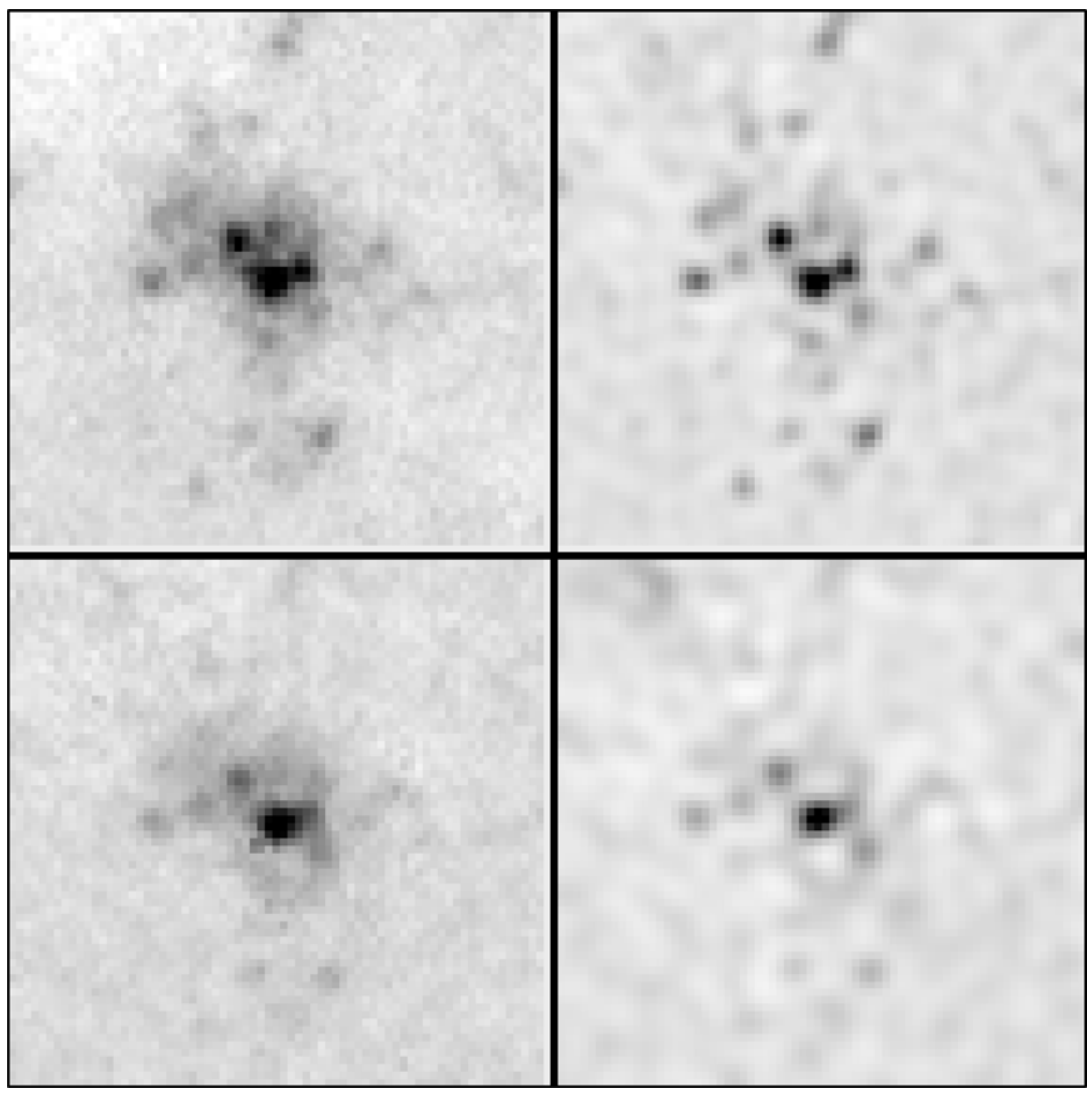

Figure 5. P3 cluster shown for $U_{330}$ (top row) and $B$ (bottom row) with the background nucleus and bulge subtracted. Images are from the reduced Nyquist-sampled images (left column) and after deconvolution (right column). The pixel scale is $0^{\prime \prime} .114$. Each image is $71 \times 71$ pixels, which corresponds to $0^{\prime \prime} 81 \times 0.0^{\prime \prime} 81$ or $3 \times 3 \mathrm{pc}$ at M31. North is at the top, and east to the left.

\subsection{Resolved Sources in the Central 1 pc of P3}

\subsubsection{Isolation of P3 from the Surrounding Nucleus}

To understand the properties of the P3 system, we attempted to isolate it from the surrounding nucleus. Since the cluster is much less prominent at $V$ and $B$ or $U_{330}$, we used the $V$ image as a proxy for the "background" light distribution for both the $B$ and $U_{330}$ images. The correct scaling required to subtract the background was somewhat problematic, given variations in color among the different nuclear stellar populations. As shown in Lauer et al. (1998), P1 is significantly redder than the outer nucleus, having $\Delta V-I=0.07$, with the portion of $\mathrm{P} 2$ outside P3 in the "anti-P1" direction having an intermediate color. A simple hypothesis is that the Tremaine disk, which comprises both $\mathrm{P} 1$ and $\mathrm{P} 2$, is redder than the outer portions of the nucleus. $\mathrm{P} 2$ appears to be slightly bluer than P1, since it has lower contrast against the underlying nucleus.

The redder color of the Tremaine disk as compared to the surrounding nucleus means that there is no simple scaling of the $V$ to the $U_{330}$ or $B$ images that will yield a completely flat background around $\mathrm{P} 3$. Scaling the $V$ image to the outer nucleus in the $U_{330}$ image oversubtracts the Tremaine disk, leaving large negative residuals at $\mathrm{P} 1$, as well as somewhat reduced but still negative residuals in the $\mathrm{P} 2$ region immediately surrounding $\mathrm{P} 3$. Conversely, scaling the two images by their P1 fluxes leaves P3 in a significantly positive background. Our solution was to scale to the portion of $\mathrm{P} 2$ immediately outside the apparent extent of the $\mathrm{P} 3$ cluster in the anti-P1 direction. This still yields a positive background in the outer nucleus, and negative residuals in P1, but does produce a low background surrounding P3. We also "clipped out" residual portion of $\mathrm{P} 3$ still present at $V$, and slightly smoothed the image as well, to avoid biasing the measurement of the central fluxes of $\mathrm{P} 3$ in $U_{330}$ or $B$, or introducing artificial structure to either image.

\subsubsection{Stellar Photometry of the P3 Sources}

The isolated P3 cluster before and after deconvolution in the $U_{330}$ and $B$ filters is shown in Figure 5. This figure zooms into the cluster, ratifying the visual impression stated at the start of this section that the outskirts of the cluster breakup into individual point sources. The deconvolved images show little diffuse emission outside the very center of the cluster. Close comparison of the $U_{330}$ and $B$ images shows that the sources cover a range in color. There are a number of sources that are readily visible in $U_{330}$, but nearly invisible in $B$ (which unfortunately give rise to large errors in the $U-B$ values of the sources). The bright source, S11, at the center of P3 is clearly extended, as is S7, the source adjacent to it; however, to the degree of resolution afforded by these observations, most of the other sources appear to be stellar.

Measuring the fluxes of the P3 sources is difficult with traditional PSF-fitting techniques, given the high degree of crowding and the strongly variable background. Instead, we used a novel procedure that measures stellar fluxes from aperture photometry on deconvolved images, which has been shown to work extremely well on Nyquist-sampled ACS/HRC images of M32 (Monachesi et al. 2011). The methodology is to take the deconvolution of the $U_{330}$ and $B$ frames to 640 Lucy-Richardson iterations. This effectively transforms the observed PSF into a highly compact "deconvolved PSF" that 


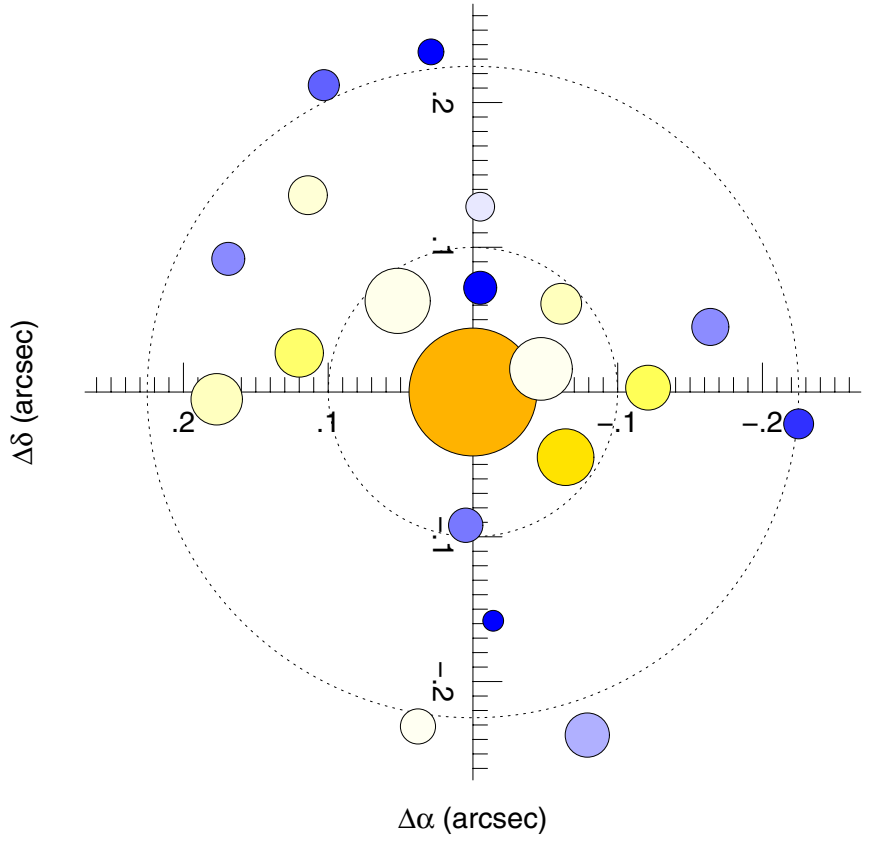

Figure 6. Symbolic "star map" of the sources in the central parsec of P3 is shown. The area of the symbols is proportional to the $B$-band luminosity. The color encodes the relative $U-B$ color of the sources. The two central sources, $\mathrm{S} 11$ and S7, are extended and are probably blends of several stars. The origin is taken as S11 in Table 1, which is inferred to mark the location of the black hole. This map can be directly compared to the images of P3 in Figure 5. The inner and outer circles correspond to orbital velocities of 1000 and $1500 \mathrm{~km} \mathrm{~s}^{-1}$ for stars in circular orbits (in the plane of the sky) about a $1.4 \times 10^{8} M_{\odot}$ black hole (Bender et al. 2005).

(A color version of this figure is available in the online journal.)

essentially concentrates all of its flux to within a radius of $00^{\prime \prime} 02$. Aperture photometry is done on the sources in the resulting image, once the background nucleus light is subtracted off. The resulting colors and magnitudes of the sources within 1 pc of P3 are mapped in Figure 6 and listed in Table 1. A comparison of this figure to the images in Figure 5 ratifies the impression that the bluer sources occur on the outskirts of the P3 cluster.

Interpreting the present photometry is complicated by the large color terms required to bring the $\mathrm{F} 330 \mathrm{~W}$ into concordance with the standard Johnson $U$ bandpass. Based on the color equations presented by Sirianni et al. (2005), for Johnson $U-B<0.2$ we derive the transformation from the F330W to $U$ bandpass as

$$
U-U_{330}=0.116-0.406(U-B)+0.125(U-B)^{2} .
$$

Fortunately, the difference between the HRC F435W filter and Johnson $B$ only varies by a few hundreds of a magnitude over the large F330W-F435W color range of the observations, and can be directly taken as $B$, given the larger random errors in the photometry. The analogous transformation for $U-B>0.2$ is

$$
U-U_{330}=0.160-0.104(U-B)-0.093(U-B)^{2} .
$$

As can be seen from the equations above, and the transformed values in Table 1, the implied corrections are as large as $\sim 40 \%$ of the observed F330W-F435W colors. While Sirianni et al. (2005) present calibrating observations over the needed range of $U-B$, the actual corrections will depend on the unknown metallicities and atmospheric properties of the actual P3 stars.

We plot the colors and absolute magnitudes of the P3 sources in Figure 7. We attempted to correct for both foreground

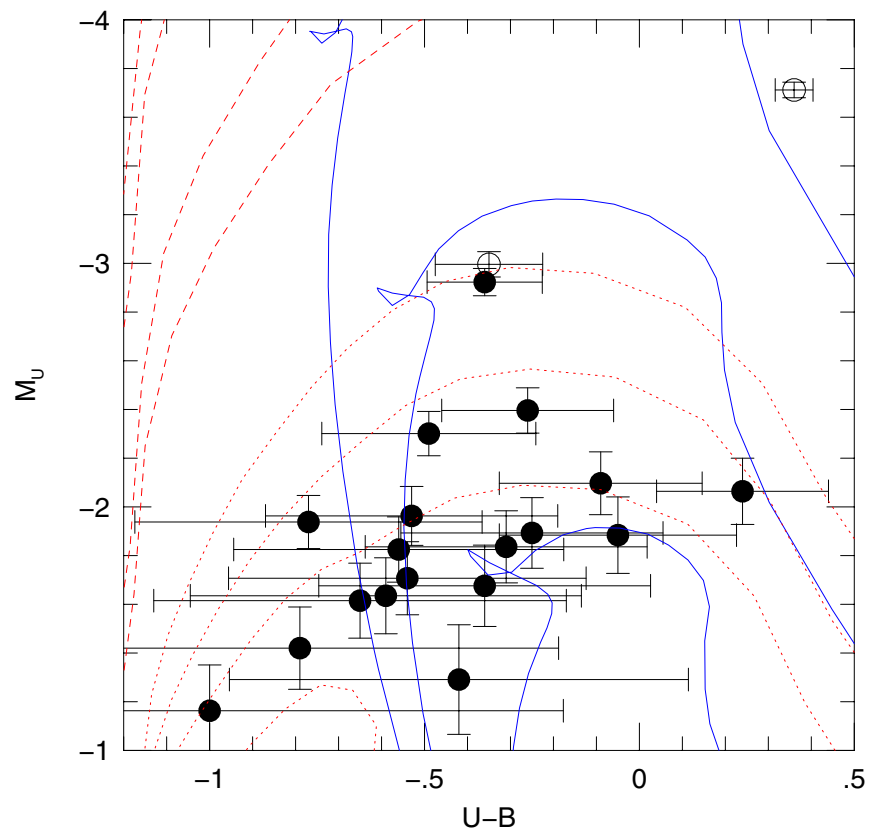

Figure 7. $U-B$ vs. $U$ color-magnitude diagram is shown for the sources within 1 pc of the P3 center. The open symbols denote S7, and S11, which are clearly spatially extended. S11, the nominal center of P3, is the brightest and reddest object plotted. Padova isochrones (Marigo et al. 2008; blue lines) are shown for single-burst populations with ages of 50, 100, and $200 \mathrm{Myr}$ going from the top down. Contrasting PAGB (red dashed lines) and PEAGB (red dotted lines) tracks (A. Bressan et al. 2012, in preparation) are shown to test the hypothesis that some of the P3 stars may really be old stars in late evolutionary phases. Going from bottom to top are one AGB-manque and three PEAGB tracks with core masses of $0.50,0.54,0.56$, and $0.58 M_{\odot}$; and four PAGB tracks are for core masses of $0.57,0.60,0.75$, and $0.90 M_{\odot}$.

(A color version of this figure is available in the online journal.)

and M31 internal dust absorption. The galactic foreground extinction provided by Schlegel et al. (1998) is $A_{V}=0.206$ mag, which given the reddening tables in Sirianni et al. (2005) yields $A_{B}=0.26$ and $A_{U_{330}}=0.31 \mathrm{mag}$. A survey of the literature shows that internal dust extinction in the M31 bulge is small (e.g., Tempel et al. 2010). Hui Dong (2011, private communication) estimates internal $A_{V}=0.08$ for the region of the bulge just outside the nucleus based on stellar population models of multi-color $H S T$ imagery. The implied total values are then $A_{B}=0.36$ and $A_{U_{330}}=0.44 \mathrm{mag}$.

Figure 7 plots Padova (Marigo et al. 2008) isochrones (blue) for single-burst stellar populations of 50, 100, and $200 \mathrm{Myr}$. It appears that most, if not all, of the sources are consistent with an age of 100-200 Myr. We note that the brightest source in the cluster, S11 in Table 1, is also the reddest source; it is extended as well. S11 is at the center of P3, and we have adopted it as the ad hoc center of the system for the coordinates given in Table 1 and Figure 6.

At the same time, evolutionary tracks for some late evolutionary phases of old stellar populations pass through the $U$ and $U-B$ domain occupied by the P3 stars, as well. These are all due to metal-rich giants that have evolved into "extreme horizontal branch" (EHB) stars and later move back to the red as their core helium-burning phase is completed. Stars that populate the EHB have lost a significant amount of their envelope on the red giant branch (RGB), though the mechanism causing the mass loss is not precisely known (for a full review see Greggio \& Renzini 1999; O'Connell 1999). These stars do not have a large enough convective envelope to allow a full 
asymptotic giant branch (AGB) phase. Instead, the hottest of these EHB stars will fail to reach the AGB track at all, and evolve through the AGB-manqué channel. Cooler EHB stars will eventually leave the AGB track early and become post early-AGB (PEAGB) stars. These phases, collectively called hot post-horizontal branch (HP-HB) evolution, have been previously found in M31 (e.g., King et al. 1995; Brown et al. 1998; Bertola et al. 1995). Their surface density has recently been shown to increase toward the center of M31 (Rosenfield et al. 2012) more rapidly than the underlying bulge surface brightness, although there appears to be no enhancement associated with the overall nucleus itself.

Figure 7 shows several examples of HP-HB tracks, such as H-burning PAGB tracks of masses $0.57<M_{\odot}<0.90$ (red, dashed) from Vassiliadis \& Wood (1994) with $Z=0.016$ and $Y=0.25$, and AGB-manqué and PEAGB tracks (red, dotted) of masses $0.50<M_{\odot}<0.58$. These are from the most recent Padova stellar evolution library (A. Bressan et al. 2012, in preparation) with $Z=0.07$ and $Y=0.389$ and an $\alpha$-enhanced composition typical of elliptical galaxies (adapted from Bensby et al. 2010). Tracks were converted to Johnson filters following the color transformations of Marigo et al. (2008). The metallicity of the Padova tracks was chosen as an extreme case to allow for high helium content, ensuring high mass loss on the RGB. As these tracks are preliminary, other metallicity and mass-loss rate combinations could also produce HP-HB stars with similar luminosities and effective temperatures.

Figure 7 shows that the canonical PAGB tracks are too bright and too hot to explain the P3 sources; however, a number of the P3 sources, especially the bluest and faintest ones, are consistent with the PEAGB evolutionary tracks shown (and the brightest of the AGB-manque tracks, which is the faintest track shown). Despite this concordance, however, we suggest that only a few of the P3 stars could be in the HP-HB phase, in contrast to being hot newly formed stars. HP-HB evolution across the $U$ and $U-B$ domain in Figure 7 is very rapid, occupying only $\sim 10^{5}$ yr. The P3 stars are highly concentrated, suggestive of a local population tightly bound to the black hole, but there is no trace of the associated substantial population of giants required to generate and maintain a short-lived UV phase sufficient to account for the complete UV luminosity of P3.

\subsection{Surface Brightness Fluctuation Analysis of the Cluster Population}

A separate approach to characterizing the stellar population of P3 is to use SBF as a diagnostic (Tonry \& Schneider 1988). In this case we are effectively using the "clumpiness" of P3 as an indicator of the typical luminosity of the stars that characterize the variance in surface brightness. For a constant total luminosity of the P3 cluster, the variance about a perfectly smooth distribution will steadily increase as we go from modeling it as the aggregate of a large number of intrinsically faint old stars to a small handful of high-luminosity young stars.

The procedure for conducting an $\mathrm{SBF}$ analysis on $\mathrm{P} 3$ requires measuring the power spectrum from the images of the cluster, and comparing it to the power spectra of models of the cluster. Measurement of the observed power spectrum begins with subtracting a smooth model of the P3 light distribution (see Section 3.4), and then measuring the power spectrum of the residual image. Since the $\mathrm{SBF}$ technique uses the shape of the PSF power spectrum as a template, the analysis is done on the background-subtracted image of P3 in the observed, as

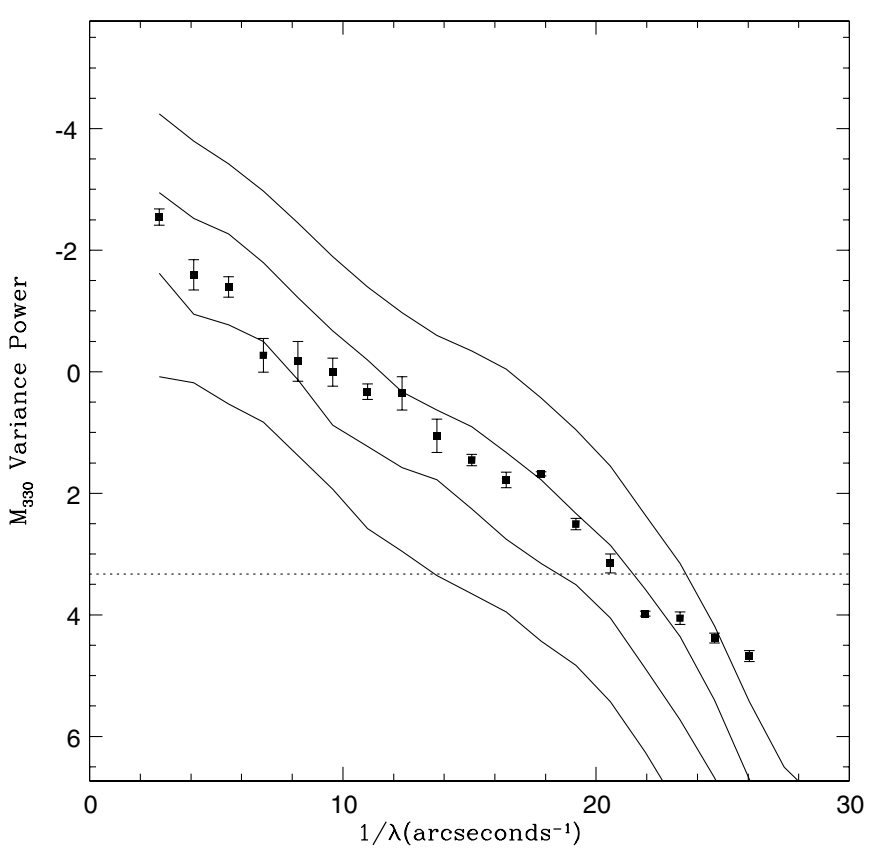

Figure 8. SBF power spectrum for the P3 cluster in the $U_{330}$ image is shown (square symbols), compared to the average power spectra for models constructed from 50, 100, 200, and $400 \mathrm{Myr}$ single-burst stellar populations (lines from top to bottom). The dotted horizontal line shows the noise level in the $U_{330}$ image. The ordinate gives the power in terms of absolute $U_{330}$ luminosity corrected for extinction. The top of the scale corresponds to the $U_{330}$ absolute magnitude of P3.

opposed to the deconvolved domain. In detail, we restricted the analysis to a $0^{\prime \prime} .5$ radius aperture centered on $\mathrm{P} 3$. We also only performed the SBF analysis on the $U_{330}$ image, given concerns about the accuracy of the technique in the $B$ image, which has a significantly reduced contribution from P3, but increased background variance (which cannot be subtracted) from the underlying old population of the nucleus. The "raw" power spectrum of $\mathrm{P} 3$ includes a constant noise term that largely represents random photon shot noise in the image. We subtracted this off by measuring the power at spatial frequencies higher than the PSF Nyquist scale. We show the resulting power spectrum in Figure 8.

The simulated power spectra come from models constructed for bursts of 50,100, 200, and $400 \mathrm{Myr}$, drawing stars at random from the Padova (Marigo et al. 2008) isochrones transformed to $U_{330}$. The location of the stars was drawn at random from the smooth model of the P3 light distribution until the total $U_{330}$ luminosity of P3 was reached. The models were then analyzed by the same procedure used for the real data. A few dozen models were generated for each burst age to average out statistical fluctuations in the power spectra. The power spectra in Figure 8 are plotted in terms of the implied absolute $U_{330}$ magnitude of the flux. The intercept of the curves at zero spatial frequency gives the luminosity of the Tonry \& Schneider (1988) $L_{*}$ parameter, which characterizes the variance as due stars of that representative brightness. The limit of the vertical scale is set by the luminosity of P3 itself.

The observed SBF power appears to fall between that of the 100 and 200 Myr bursts, which is qualitatively ratified by the subjective appearance of the P3 models of the same age. This result is thus in excellent agreement with the P3 color-magnitude diagram (CMD) analysis presented in the previous section. 

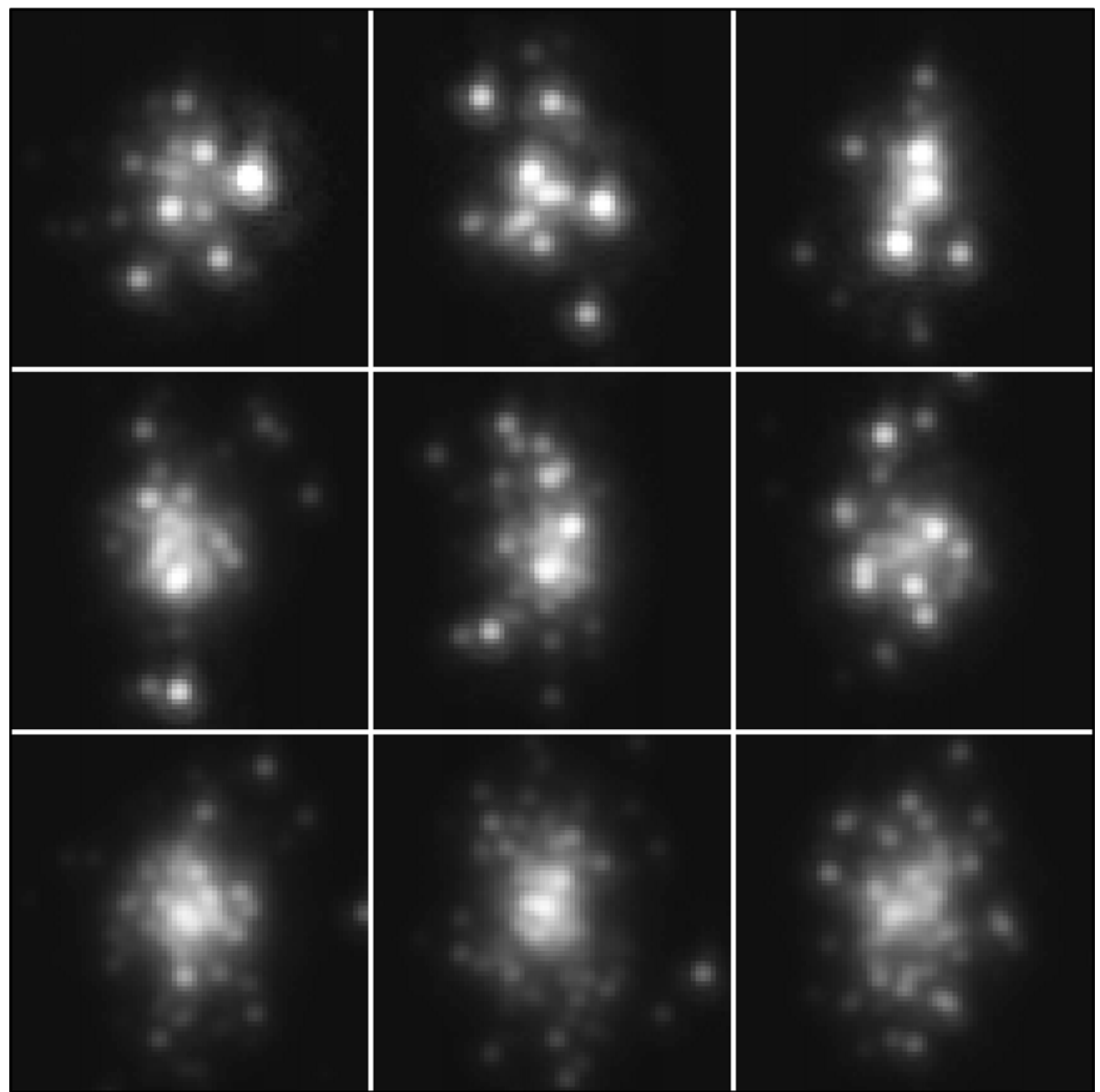

Figure 9. Models of the $\mathrm{P} 3$ cluster based on the smooth light distribution derived in Section 3.4 and random samples of stars drawn from the Padova isochrones (Marigo et al. 2008) for bursts of $50 \mathrm{Myr}$ (top row), $100 \mathrm{Myr}$ (middle), and $200 \mathrm{Myr}$ (bottom). Each row shows three different realizations for the given burst age. The scale and image size are the same as in Figure 5.

While we can constrain the age of the $\mathrm{P} 3$ population, it appears, however, that we have little sensitivity to the form of the initial mass function (IMF), given the limited dynamic range of the luminosity of the sources seen in P3. Bartko et al. (2010) argue that the stars surrounding the Milky Way black hole have a markedly "top-heavy" IMF. We generated $200 \mathrm{Myr}$ SBF models with their $d N / d m \propto m^{-0.5}$ IMF to see if this might produce significantly different SBF power as compared to that for the same age derived from the Padova isochrones. We only measured a modest increase in SBF power, however, which would cause us to infer a slightly older age for the population by $\sim 50 \mathrm{Myr}$ (it takes an older "top-heavy" model to match the observed SBF power of P3 itself), a result that is within the error limits of the SBF results already quoted. The SBF power is dominated by the resolved sources near the MS turnoff, which is determined by age alone. A more top-heavy IMF qualitatively down-weights the contribution of fainter stars on the MS to the total SBF power, causing the relative SBF variance to increase for the same age and total P3 luminosity. However, given the steep $L \sim m^{4}$ mass-luminosity relation for the MS, we have in the end little leverage on the mass function. A small range in MS mass corresponds to a large change in stellar luminosity, thus suppressing information on the mass function itself. We further note that the CMD analysis has even less sensitivity to the IMF, given the even more limited mass range of the resolved stars.
Some sample models for P3 are shown in Figure 9. The clumpiness of the models shown can be seen to steadily decrease with increasing burst age. It is notable that the strong SBF pattern completely dominates the appearance of the simulated clusters. Further, the location of either the peak brightness or light-weighted centroid of the entire cluster can be displaced significantly away from the nominal center of the ideal smooth cluster model due to the large random fluctuations in the structure. This is an important caveat to respect when evaluating the precise location of the $\mathrm{P} 3$ cluster with respect to the M31 center of mass or the location of the M31 black hole (see below). For the ensemble of $50 \mathrm{Myr}$ models, the average location of the peak flux is offset from the true cluster center by 0.12 , and the photo-center is displaced placed by $00^{\prime} 05$, with the typical scatter from model to model being nearly as large as these mean values. For the 100 Myr models, the two offsets decrease to 0'.06 and 0.02; and 0.03 and 0.01 for the 200 Myr models.

\subsection{The Spectral Energy Distribution of P3}

The total brightness of $\mathrm{P} 3$ was measured by aperture photometry performed on the difference images. The apparent magnitudes of $\mathrm{P} 3$ are $20.3 \pm 0.1(\mathrm{AB})$ or $19.1 \pm 0.1$ (Vega) in $U_{330}$, and $19.0 \pm 0.1(\mathrm{AB})$ and $19.1 \pm 0.1$ (Vega) in $B$, uncorrected for foreground extinction. Using the reddening values stated 


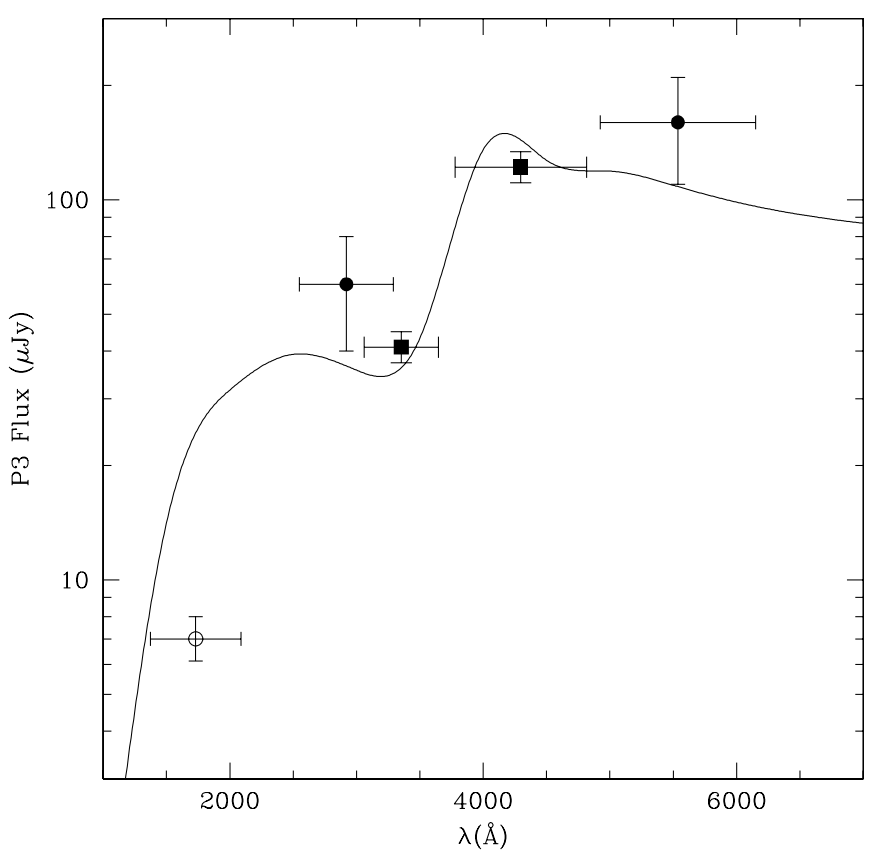

Figure 10. SED of $P 3$ is shown based on the broadband total magnitudes of the present study (large squares), Lauer et al. (1998; round dots), and the FUV flux of King et al. (1995; open circle). The horizontal bars give the approximate bandwidths. An $f_{v}$ SED template for an A0 V star is shown scaled to the points for comparison.

earlier, the corrected apparent fluxes are $42 \pm 4 \mu \mathrm{Jy}$ in $U_{330}$ and $125 \pm 12 \mu \mathrm{Jy}$ in $B$. The implied reddening-corrected colors are $U_{330}-B=-0.1 \pm 0.2$ and $B-V=0.4 \pm 0.3$ (Vega), using the $m_{V}$ measure from Lauer et al. (1998).

The implied SED of P3 is plotted in Figure 10, based on the FUV $175 \mathrm{~nm}$ flux measured by King et al. (1995), the $U_{330}$ and $V$ fluxes measured by Lauer et al. (1998), and the present $U_{330}$ and $B$ values. We reduced the FUV measurement from $6.7 \mu \mathrm{Jy}$ to $5.6 \mu \mathrm{Jy}$, for consistency with the present reddening corrections. King et al. (1995) assumed $A_{175}=0.88$, based on the total $E(B-V)=0.11$ for M31 measured by McClure \& Racine (1969). Our $E(B-V)=0.086$ value implies $A_{175}=0.69$, instead. An $f_{v}$ SED of an A0 MS star scaled to the flux points is shown for comparison. As noted in Lauer et al. (1998), and shown spectroscopically by Bender et al. (2005), the SED of P3 is indeed "A-like." The FUV point does fall well below the template, but this portion of the SED is highly sensitive to temperature, and the FUV point could be fitted with a modest adjustment of the template; this point will also be the most sensitive to the correct value of the extinction.

\subsection{The Surface Brightness Profile of P3}

The overall distribution of light within the P3 cluster is confirmed to roughly follow the exponential light distribution derived by Bender et al. (2005). A $U_{330}$-band surface brightness profile of P3 is shown in Figure 11, with the exponential model shown as a solid line. S11 was taken as the center of the cluster, but the fit excludes points with $r<0$ '.04, given that the innermost points will still be affected by the residual PSF associated with the deconvolution. The exponential form recovered is

$$
\mu_{330}(a)=(13.4 \pm 1.7) a+16.45 \pm 0.32,
$$

where $a$ is the semimajor axis in arcseconds, and $\mu_{330}$ is the surface brightness (mag $\operatorname{arcsec}^{-2}$ ) observed with the F330W

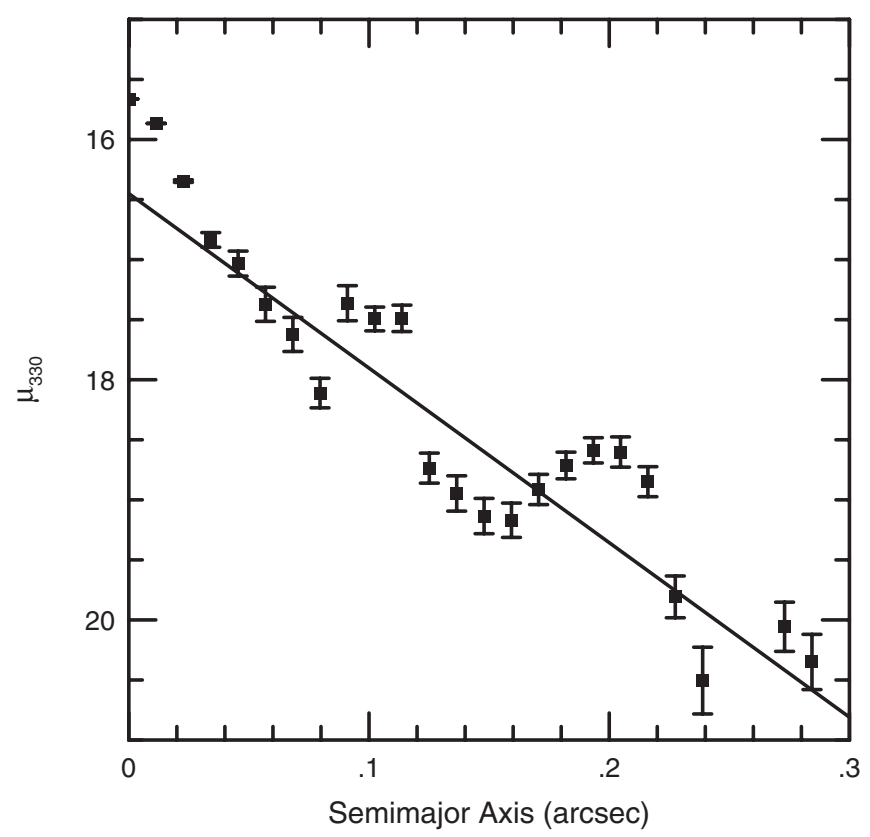

Figure 11. Apparent surface brightness of the $\mathrm{P} 3$ cluster in $U_{330}$ as a function of semimajor axis is plotted. The solid line shows the exponential form fitted to the profile for $r>0^{\prime \prime} .04$.

Vega-based zero point (no reddening correction has been applied). The implied exponential scale length is $00^{\prime} 075 \pm 0.010$, which is marginally more compact than the $00^{\prime \prime} 1 \pm 0.01$ value derived by Bender et al. (2005). The average ellipticity of the isophotes is $0.33 \pm 0.03$, which implies an inclination of $48^{\circ} \pm 5^{\circ}$, if P3 is interpreted to be a disk, again in good agreement with the $55^{\circ} \pm 2^{\circ}$ of Bender et al. (2005).

Although S11 was excluded from the fit, given concerns about the residual deconvolved PSF, the brightness profile of S11 actually rises about the exponential model. The core radius of $\mathrm{S} 11$ is $<0^{\prime} .024$ or $<0.09 \mathrm{pc}$. If we estimate the volume luminosity density of $\mathrm{S} 11$ from $\rho_{L}=I_{0} /\left(2 r_{C}\right)$, where $I_{0}$ is the central surface brightness $(<15.53$ in $U$, corrected for extinction), then at $\mathrm{S} 11$ we find $\rho_{L}>3 \times 10^{5} L_{\odot} \mathrm{pc}^{-3}$ in the $U$ band.

\subsection{The Location of the M31 Black Hole}

The present work provides no new dynamical information about the M31 black hole, and cannot offer any objective improvement on the conclusion of Kormendy \& Bender (1999) or Bender et al. (2005) that the black hole must be close to the center of P3. At the same time, we advance subjective arguments that the location of the black hole may be coincident with S11 (see Table 1). The central location of this source is suggestive. While we showed in Section 3.2 that simulated models of the cluster often have the brightest clump of stars to be significantly offset from the true center of the cluster (which is where we would expect the black hole to be). However, while we do not know the "true" center of the P3 case, the location of S11 and the photo-center of P3 at least agree to within 0.'02. S11 is also extended and significantly redder than the other sources, underscoring its unique properties among the other sources. Its red color indeed makes it the only source to mark the location of P3 in the $V$ band. A truly extended source should not exist within the strong tidal field of the black hole, unless it represents the peak of a cusp made of the stars most closely bound to it. At the same time, it is possible that the structure of S11 is only 
due to a random fluctuation of stars along the line of sight. S07 is also extended, is offset from the center of P3, but otherwise has no special attributes. The SBF simulations presented in the previous section show that most of the sources in P3 are indeed dominated by a single bright star; however, very close to the center there can be one or two blends of similarly bright stars within the HRC PSF.

\section{DISCUSSION, SUMMARY, AND CONCLUSION}

We began the paper with a history of earlier work on P3, which already built a strong case that it is a cluster of young stars. We have now partially resolved the cluster into individual stars and characterized its form with significantly higher spatial resolution than was provided by the WFPC2/PC observations of Lauer et al. (1998); however, in broad detail the conclusions of that paper and the later work of Bender et al. (2005) remain unaltered. Both a CMD obtained of the resolved stars and an SBF analysis performed on the total image show that $\mathrm{P} 3$ plausibly comprises a population formed in a burst 100-200 Myr ago, consistent with the P3 SED and the spectrum obtained by Bender et al. (2005). The result may also be supported by the Saglia et al. (2010) spectroscopy observation of the inner bulge, which suggests that a burst of star formation occurred within the nucleus about $\sim 100$ Myr ago.

One small modification of the earlier results provided by the present work is that P3 may be slightly younger than the $200 \mathrm{Myr}$ age derived by Bender et al. (2005), but that work really only ruled out an age much younger than 100 Myr. Another new result is that the color of S11 suggests that a single-age starburst model for the cluster does not capture the full story of P3. At some level, the large luminosity of P3 blends with the much bluer stars around it to generate an overall A-like SED. It is intriguing that $\mathrm{S} 11$ provides the only visible trace of $\mathrm{P} 3$ in the $V$ band. If it does mark the location of the M31 black hole, then it suggests that there may yet be another change in the stellar population of the nucleus within $\sim 0{ }^{\prime} 03$ or $\sim 0.1 \mathrm{pc}$ of the black hole.

Although we have concluded that the P3 stars are young, King et al. (1995) and Brown et al. (1998) argue that the UV-bright stars in the bulge of M31, which of course surrounds the nucleus, represent the final stages of stellar evolution in an old metal-rich stellar population. If there are truly two different populations contributing UV-bright stars to the bulge and nucleus of M31, it still begs the question of whether or not old remnants may be present in P3, as well as how far out the putative young stars in P3 may be traced out into the surrounding nucleus or even bulge. We show that PEAGB tracks traverse the $U$ versus $U-B$ domain defined by the P3 stars, thus it is possible that a few of the P3 stars are indeed old-evolved stars, rather than being young massive MS stars. However, since this is a very rapid evolutionary phase, it is extremely unlikely that the entire P3 cluster could be accounted for by PEAGB stars.

A strong factor in constraining the population of P3 is the compactness of P3 itself. The UV-stars outside the nucleus do trace the bulge light at long wavelengths, but yet are not strongly concentrated within the outer nucleus; their abundance by total light or number is but a small fraction of the bulge. Requiring any sort of HP-HB stars as the dominant P3 population requires an associated massive population of red giants also closely bound to the M31 black hole to continually generate the short-lived PEAGB stars. There is no evidence for such a population in the $V$ and $I$ nuclear images of Lauer et al. (1998).
This same consideration would appear to also apply to the hypothesis of Demarque \& Virani (2007), who suggest that the P3 UV-bright stars may be the remnant cores of giants stripped by close encounters with the black hole. The high velocities associated with the black hole are such that stars passing close to the black hole from the bulge or even the outer nucleus will not dwell in the vicinity of P3. If the P3 stars are really processed giants, they still must have been initially closely bound to the black hole, which again begs the question of where the progenitor giants are. The only way that this mechanism might work is if the stripping of the P3 stars takes place just as they begin their first ascent up the red giant branch. This might produce long-lived UV-bright stripped cores, and would account for lack of progenitor giants as well.

Further investigation of the origin of P3 might be linked to understanding the early-type stars closely bound to the black hole in our Galaxy. The existence of P3 in a second Local Group galaxy suggests that this is not a rare phenomenon. The present observations offer an additional site to test mechanisms that can form stars within the strong tidal field of a supermassive black hole. We finish by noting that as with the galactic center, it may be possible to measure the proper motions of stars closely bound to the M31 black hole. Figure 6 shows that circular velocities in the plane of the sky around the M31 black hole will exceed $1000 \mathrm{~km} \mathrm{~s}^{-1}$ over the entire extent of P3. It is possible that proper motions may be detected in about a decade after the present observations were made.

We thank Karl Gebhardt, Scott Tremaine, Jessica Lu, and Pierre Demarque for useful conversations. We thank Alessandro Bressan and Leo Girardi for advance use of preliminary Padova stellar evolution tracks. We thank the referee for a number of useful suggestions. Pete Merenfeld (NOAO) kindly prepared the three-color image of the nucleus. The National Optical Astronomy Observatory is operated by AURA, Inc., under cooperative agreement with the National Science Foundation.

\section{REFERENCES}

Alexander, T. 2006, J. Phys.: Conf. Ser., 54, 243

Allen, D. A., Hyland, A. R., \& Hillier, D. J. 1990, MNRAS, 244, 706

Bartko, H., Martins, F., Trippe, S., et al. 2010, ApJ, 708, 834

Bender, R., Kormendy, J., Bower, G., et al. 2005, ApJ, 631, 280

Bensby, T., Feltzing, S., Johnson, J. A., et al. 2010, A\&A, 512, A41

Bertola, F., Bressan, A., Burstein, D., et al. 1995, ApJ, 438, 680

Bonnell, I. A., \& Rice, W. K. M. 2008, Science, 321, 1060

Brown, T. M., Ferguson, H. C., Stanford, S. A., \& Deharveng, J.-M. 1998, ApJ, 504, 113

Chang, P., Murray-Clay, R., Chiang, E., \& Quataert, E. 2007, ApJ, 668, 236

Crane, P. C., Dickel, J. R., \& Cowan, J. J. 1992, ApJ, 390, L9

del Burgo, C., Mediavilla, E., \& Arribas, S. 2000, ApJ, 540, 741

Demarque, P., \& Virani, S. 2007, A\&A, 461, 651

Dressler, A. 1984, ApJ, 286, 97

Dressler, A., \& Richstone, D. O. 1988, ApJ, 324, 701

Forrest, W. J., Shure, M. A., Pipher, J. L., \& Woodward, C. E. 1987, in AIP Conf. Proc. 155, The Galactic Center, ed. D. A. Backer (Melville, NY: AIP), 153

Freedman, W. L., \& Madore, B. F. 1990, ApJ, 365, 186

Fruchter, A. S., \& Hook, R. N. 2002, PASP, 114, 144

Garcia, M. R., Hextall, R., Baganoff, F. K., et al. 2010, ApJ, 710, 755

Garcia, M. R., Williams, B. F., Yuan, F., et al. 2005, ApJ, 632, 1042

Greggio, L., \& Renzini, A. 1999, Mem. Soc. Astron. Ital., 70, 691

King, I. R., Deharveng, J. M., Albrecht, R., et al. 1992, ApJ, 397, L35

King, I. R., Stanford, S. A., \& Crane, P. 1995, AJ, 109, 164

Kormendy, J. 1988, ApJ, 325, 128

Kormendy, J., \& Bender, R. 1999, ApJ, 522, 772

Krabbe, A., Genzel, R., Eckart, A., et al. 1995, ApJ, 447, L95

Lauer, T. R. 1999, PASP, 111, 227 
Lauer, T. R., Faber, S. M., Ajhar, E. A., Grillmair, C. J., \& Scowen, P. A. 1998, AJ, 116, 2263

Lauer, T. R., Faber, S. M., Groth, E. J., et al. 1993, AJ, 106, 1436

Levin, Y., \& Beloborodov, A. M. 2003, ApJ, 590, L33

Li, Z., Wang, Q. D., \& Wakker, B. P. 2009, MNRAS, 397, 148

Lucy, L. B. 1974, AJ, 79, 745

Marigo, P., Girardi, L., Bressan, A., et al. 2008, A\&A, 482, 883

McClure, R. D., \& Racine, R. 1969, AJ, 74, 1000

Monachesi, A., Trager, S. C., Lauer, T. R., et al. 2011, ApJ, 727, 55

Nieto, J.-L., Macchetto, F. D., Perryman, M. A. C., di Serego Alighieri, S., \& Lelievre, G. 1986, A\&A, 165, 189

O'Connell, R. W. 1999, ARA\&A, 37, 603

Peiris, H. V., \& Tremaine, S. 2003, ApJ, 599, 237
Redman, R. O., \& Shirley, E. G. 1937, MNRAS, 97, 416

Richardson, W. H. 1972, J. Opt. Soc. Am., 62, 52

Richstone, D., Bower, G., \& Dressler, A. 1990, ApJ, 353, 118

Rosenfield, P., Johnson, L. C., Girardi, L., et al. 2012, ApJ, submitted

Saglia, R. P., Fabricius, M., Bender, R., et al. 2010, A\&A, 509, A61

Schlegel, D. J., Finkbeiner, D. P., \& Davis, M. 1998, ApJ, 500, 525

Sirianni, M., Jee, M. J., Benítez, N., et al. 2005, PASP, 117, 1049

Tempel, E., Tamm, A., \& Tenjes, P. 2010, A\&A, 509, A91

Tonry, J., \& Schneider, D. P. 1988, AJ, 96, 807

Tremaine, S. 1995, AJ, 110, 628

Vassiliadis, E., \& Wood, P. R. 1994, ApJS, 92, 125

Wardle, M., \& Yusef-Zadeh, F. 2012, ApJ, submitted (arXiv:1108.2175) 